\title{
Can You BET on PET in Cancer?
}

\author{
Chintamani $^{1}$
}

Received: 21 March 2016 / Accepted: 21 March 2016 / Published online: 4 April 2016

(C) Association of Surgeons of India 2016

All that glitters on PET is not cancer and all that does not is not benign. Positron emission tomography or PET scan is perhaps one of the most misused investigations in the field of oncology. Although theoretically the concept is very appealing as that of a stand-alone test that not only localizes the primary disease but also picks up metastases, there are some serious pitfalls that need to be considered [1].

Cost being a major factor, the test is not freely and easily available even in the developed world. With better availability and increasing prosperity in developing countries like India, it has become a common site, especially in the metros to find a patient walking in with a PET film during his/her first visit to a physician. The scene is scary as it may take away from this wonderful tool its real utility in diagnosing and accurately staging/ prognosticating cancer.

In the developing world, there is an additional problem with the burden of infectious diseases like tuberculosis where PET may pick up false positives as the FDG uptake is non-specific in inflammations $[2,3]$. The cancer in certain cases may be upstaged and PET may actually prevent organ and function preservation by falsely making a potentially curable disease appear metastatic. Some of these lesions (nodes) may be in inaccessible regions like mediastinum, thus forcing an oncologist to chase these foci fruitlessly and endlessly

Chintamani

drchintamani7@gmail.com

1 VMMC \& Safdarjang Hospital, New Delhi, India causing significant delays in definitive management of cancer.

Needless to say, it is one test that has a very significant role in the assessment and management of recurrent or remnant cancer, and this continues to remain its most common indication. Also in the management of certain cancers like medullary thyroid cancer (MTC), PET scan has a great role in localizing the primary disease, recurrence, and metastases as these cancers do not pick up iodine. Therefore, a commonly used phrase in MTC is RET for some \& PET for all.

Another very significant utility of this test is in assessing anatomical and response to neo-adjuvant chemo/radio therapy, which should ideally be more reliable. Response Evaluation Criteria In Solid Tumors (PET-RECIST) has been used in assessing response to neo-adjuvant chemotherapy in various cancers like soft tissue sarcomas [1].

Although the test has now been included in the latest National Cancer and Comprehensive Network (NCCN) guidelines for staging and management of certain cancers, one has to be careful in preventing its misuse. Therefore, there is a need to educate both the physicians and patients about false positives and the limitations of PET scan. The test also needs a trained and dedicated nuclear medicine expert for its accurate interpretation, as there may be subjective variations $[2,3]$.

There are certain instances of physiological aberrations that need to be interpreted carefully. For example, since the isotope is excreted through urinary tract, the lower ureters may provide a false impression of increased activity besides the brown fat related activity and also the pick up by salivary glands in the head and neck regions. The test is, however, invaluable if tailored correctly to the patient, cancer, and the therapy. 


\section{References}

1. Chintamani (2011) Soft tissue sarcomas-the pitfalls in diagnosis and management. Ind J Surg Oncol 2(4):261-4
2. Shreve PD, Anzai Y, Wahl RL (1999) Pitfalls in oncologic diagnosis with FDG PET imaging: physiologic and benign variants. Radiographics 19(1):61-77, 150-1

3. Cook GJ (2007) Pitfalls in PET/CT interpretation. Q J Nucl Med Mol Imaging 51(3):235-243 